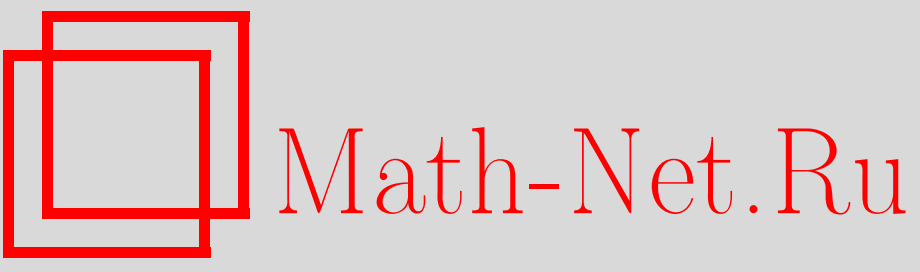

А. А. Феликсон, О сигнатурах Терстона, УМH, 1997, том 52, выпуск 4, 217-218

DOI: https://doi.org/10.4213/rm877

Использование Общероссийского математического портала Math-Net.Ru подразумевает, что вы прочитали и согласны с пользовательским соглашением

http://www.mathnet.ru/rus/agreement

Параметры загрузки:

IP: 3.89 .185 .249

26 апреля 2023 г., 18:15:35 


\section{О СИГНАТУРАХ ТЕРСТОНА}

\section{А. А. ФЕликСОН}

В препринте [4] В. Терстон рассмотрел пространство $E\left(a_{1}, \ldots, a_{n}\right)$ таких евклидовых метрик на сфере $S^{2}$, у которых особенности являются коническими точками с заданньми кривизнами $2 \pi a_{i}, 0<a_{i}<1, i=1, \ldots, n, n>3$. Числа $a_{i}$ назовем сигнатурой евклидовой метрики $c$ особенностями.

Хорошо известно, что $\sum a_{i}=2$. Нормируя метрики с данной сигнатурой условием равенства единице площади сферы, В. Терстон сформулировал условия на сигнатуру, при которых пространство $E\left(a_{1}, \ldots, a_{n}\right)$ оказывается комплексным гиперболическим орбифолдом конечного объема, т.е. фактор-пространством $B^{n-3} / \Gamma$ комплексного шара $B^{n-3}$ по дискретной решетке его автоморфизмов. Эти условия таковы:

(1) $a_{i} \in \mathbb{Q}, 0<a_{i}<1, i=1, \ldots, n,\left(a_{i}=p_{i} / q_{i}, p_{i}<q_{i} \in \mathbb{N}\right)$;

(2) $\sum_{i=1}^{n} a_{i}=2$

(3) $a_{i}+a_{j}<1, a_{i} \neq a_{j} \Rightarrow \frac{1}{1-a_{i}-a_{j}} \in \mathbb{Z}$ (т.е. $\left.a_{i}+a_{j}=1-\frac{1}{k_{i j}}, k_{i j} \in \mathbb{Z}\right)$;

(4) $a_{i}+a_{j}<1, a_{i}=a_{j}, i \neq j \Rightarrow \frac{1}{1-a_{i}-a_{j}} \in \frac{1}{2} \mathbb{Z}$ (т.е. $a_{i}+a_{j}=\frac{1}{2}-\frac{1}{k_{i j}}, k_{i j} \in \mathbb{Z}$ ).

Возникает естественная задача нахождения всех таких сигнатур. Для $n=4$ существует бесконечное число сигнатур Терстона (см. [2], [4]). Для $n \geqslant 5$ список таких сигнатур конечен. Он впервые появился у Мостова в [3], но был неполон. Потом он был уточнен Терстоном, сделавшим полньй компьютерньй перебор для наборов $a_{i}$, в которых наименьший общий знаменатель не превосходит 256. Но не было доказано, что нет таких сигнатур с общим знаменателем, большим 256. Основной результат данной работы - доказательство их отсутстия, основанное на элементарных теоретико-числовых соображениях.

Автор пользуется случаем выразить благодарность О.В. Шварцману.

Обозначения. Перепишем условие (2), сгруппировав равные слагаемые и расставив их по возрастанию:

$$
l_{1} a_{1}+\cdots+l_{r} a_{r}+a_{r+1}+\cdots+a_{r+s}=2,
$$

где $a_{i} \neq a_{j}$ при $i \neq j ; l_{i}>1, l_{i} \in \mathbb{N} ; a_{1}<a_{2}<\cdots<a_{r} ; a_{r+1}<\cdots<a_{r+s}$.

Все буквы, кроме $a_{i}$, в дальнейшем означают натуральные числа.

Ограниченность числа слагаемых.

ЛЕмма 1. Пусть $a_{1}<a_{2}<a_{3}$ и набор $a_{i}$ является решением задачи. Тогда либо $a_{1}+a_{2}+a_{3}>1$, либо $\left(a_{1}, a_{2}, a_{3}\right)=\left(\frac{1}{2}, \frac{1}{3}, \frac{1}{6}\right)$ u $q_{i} \leqslant 6$ (а значит, $\left.n \leqslant 12\right)$.

ДокАЗАТЕльство. Пусть $a_{1}, a_{2}, a_{3}$ - наименьшие различные числа в наборе. Пусть $a_{1}+$ $a_{2}+a_{3} \leqslant 1$. Тогда $a_{i}+a_{j}<1$ (при $\left.i, j \in\{1,2,3\}, i \neq j\right) \Rightarrow a_{1}+a_{2}=1-\frac{1}{k_{1}}, a_{1}+a_{3}=1-\frac{1}{k_{2}}$, $a_{2}+a_{3}=1-\frac{1}{k_{3}}$.

Так как $a_{1}+a_{2}<a_{1}+a_{3}<a_{2}+a_{3}$, то $k_{1}<k_{2}<k_{3}$.

Так как $\left(a_{1}+a_{2}\right)+\left(a_{2}+a_{3}\right)+\left(a_{3}+a_{1}\right) \leqslant 2$, то $3-\frac{1}{k_{1}}-\frac{1}{k_{2}}-\frac{1}{k_{3}} \leqslant 2$, т.е. $1 \leqslant \frac{1}{k_{1}}+\frac{1}{k_{2}}+\frac{1}{k_{3}}$.

Так как $k_{1}<k_{2}<k_{3}$, то тройка $\left(k_{1}, k_{2}, k_{3}\right)$ имеет одно из следующих значений: а) $(2,3,4)$, b) $(2,3,5)$, c) $(2,3,6)$.

Выражая $\left(a_{1}, a_{2}, a_{3}\right)$ через $\left(k_{1}, k_{2}, k_{3}\right)$, получаем в случае а) $\left(\frac{5}{24}, \frac{7}{24}, \frac{11}{24}\right)$, в случае b) $\left(\frac{11}{60}, \frac{19}{60}, \frac{29}{60}\right)$, в случае с) $\left(\frac{1}{6}, \frac{1}{3}, \frac{1}{2}\right)$.

В случаях а) и b) по условию (4) $l_{1}=1$ и $l_{2}=1$. Значит, $a_{5} \geqslant a_{4} \geqslant a_{3}$. В случае а) $a_{5}<2-$ $\left(a_{1}+a_{2}+2 a_{3}\right)=\frac{14}{24}<a_{3}-$ противоречие.

В случае b) проверкой условий (1)-(4) убеждаемся, что $a_{4} \neq a_{3}$. Но тогда $a_{1}+a_{2}+a_{4}>1$ (поскольку иначе $a_{1}+a_{4}=\frac{2}{3}=a_{1}+a_{3}$ ), т.е. $a_{3}+a_{5}=1-\frac{1}{m}, a_{2}+a_{5}=1-\frac{1}{k}, a_{1}+a_{5}=1-\frac{1}{p}$ $\Rightarrow a_{5}=\frac{31}{60}-\frac{1}{m}=\frac{41}{60}-\frac{1}{k}=\frac{49}{60}-\frac{1}{p} \Rightarrow \frac{2}{15}=\frac{1}{p}-\frac{1}{k}, \frac{1}{6}=\frac{1}{k}-\frac{1}{m} \Rightarrow k \leqslant 6 \Rightarrow k=5$ (иначе не удовлетворится уравнение $\left.\frac{2}{15}=\frac{1}{p}-\frac{1}{k}\right) \Rightarrow a_{5}=\frac{29}{60}=a_{3}$ - противоречие.

В случае с) надо только показать, что $r+s \leqslant 4$ (тогда $a_{4}=2-\sum_{i=1}^{3} l_{i} a_{i}$ и $q_{4} \leqslant 6$ ). Действительно, если $a_{1}<a_{2}<a_{3}<a_{4}<a_{5}$, то $a_{4}+a_{5}>1$ и $\sum a_{i}>2$. 
ЗАмечАниЕ 1 . В дальнейшем можно считать, что $a_{1}<a_{2}<a_{3} \Rightarrow a_{1}+a_{2}+a_{3}>1$.

ЛЕМма $2 . r \leqslant 2, \sum_{i=1}^{r} l_{i} \leqslant 12$.

ДокАЗАТЕЛЬСТво. Пусть $r \geqslant 3$. Тогда $(2) \Rightarrow a_{1}+a_{2}+a_{3} \leqslant 1$, что невозможно по лемме 1 (с учетом замечания). Значит, $r<3$.

Так как при $i \leqslant r$ либо $a_{i} \geqslant \frac{1}{2}$, либо $a_{i}=\frac{1}{2}-\frac{1}{k} \geqslant \frac{1}{2}-\frac{1}{3}=\frac{1}{6}$, то $\sum_{i=1}^{r} l_{i} \leqslant 12$.

ЛЕмма 3. $r+s<6$.

ДокАЗАТЕЛЬСтво. Пусть $r+s \geqslant 6$. По лемме $1 a_{1}+a_{2}+a_{3}>1$ и $a_{4}+a_{5}+a_{6}>1$, что противоречит тому, что $\sum_{i=1}^{n} a_{i}=2$.

Ограниченность знаменателей.

Для каждого из возможных значений $r+s$ надо показать, что $q_{i}<200$. Метод доказательства продемонстрируем на наиболее простом случае $-r+s=5$.

ЛЕмма 4 (вспомогательная). Пусть $\frac{1}{2}=\frac{1}{k}+\frac{1}{l}+\frac{1}{m}$ uлu $\frac{1}{3}=\frac{1}{k}+\frac{1}{l}+\frac{1}{m}$.

1) Пусть $\frac{1}{2}=\frac{1}{k}+\frac{1}{\tau}+\frac{1}{m}$. Тогда с точностью до перестановки тройка $(k, l, m)$ принимает одно из следующих значений: $(6,6,6),(5,5,10),(4,8,8),(4,6,12),(4,5,20)$, $(3,12,12),(3,10,15),(3,9,18),(3,8,24),(3,7,42)$.

2) Пусть $\frac{1}{3}=\frac{1}{k}+\frac{1}{l}+\frac{1}{m} u k<l<m$. Тогда тройка $(k, l, m)$ принимает одно из следующих значений: $(6,10,15),(6,9,18),(6,8,24),(6,7,42),(5,12,20),(5,10,30)$, $(5,9,45),(5,8,120),(4,21,28),(4,20,30),(4,18,36),(4,16,48),(4,16,60),(4,14,84)$, $(4,13,156)$.

ДокаЗАтЕльство. Докажем первое утверждение леммы. Пусть $k \leqslant l \leqslant m$. Тогда $\frac{1}{6} \leqslant$ $\frac{1}{k} \leqslant \frac{1}{3}$

При каждом допустимом значении $k$ имеем $\frac{1}{k} \geqslant \frac{1}{\tau} \geqslant \frac{1}{2}\left(\frac{1}{2}-\frac{1}{k}\right)$. Проверяя для всех допустимых пар $(k, l)$ (их конечное число) условие $\frac{1}{2}-\frac{1}{k}-\frac{1}{l}=\frac{1}{m}$, находим все решения.

Второе утверждение леммы доказьвается аналогично.

Лемма 5. Пусть $r+s=5$. Тогда $q_{i} \leqslant 84$.

ДоКАЗАТЕЛЬСтвО. Пусть $a_{1}<a_{2}<a_{3}<a_{4}<a_{5}$. Пусть $r>0$. Тогда по лемме 1 $a_{1}+a_{2}+a_{3}>1$ и $a_{1}+a_{4}+a_{5}>1$, что противоречит тому, что $\sum_{i=1}^{n} a_{i}=2$. Значит, $r=0$.

Так как сумма любых трех различных слагаемых болшше единицы, то сумма двух оставшихся - меньше единицы. Пусть $a_{1}+a_{2}=1-\frac{1}{k}, a_{3}+a_{4}=1-\frac{1}{m}, a_{3}+a_{5}=1-\frac{1}{u}, a_{4}+a_{5}=1-\frac{1}{\tau}$, $a_{1}+a_{3}=1-\frac{1}{w}, a_{2}+a_{4}=1-\frac{1}{t}, a_{2}+a_{5}=1-\frac{1}{p}, a_{4}+a_{5}=1-\frac{1}{\tau}$. Тогда $\frac{1}{k}>\frac{1}{m}>\frac{1}{u}>\frac{1}{\tau}$ и $\frac{1}{k}>\frac{1}{w}>\frac{1}{t}>\frac{1}{p}>\frac{1}{l}$.

Так как $4=2\left(a_{1}+a_{2}\right)+\left(a_{3}+a_{4}\right)+\left(a_{3}+a_{5}\right)+\left(a_{4}+a_{5}\right)$, то $1=\frac{2}{k}+\frac{1}{m}+\frac{1}{u}+\frac{1}{l}$. Так как $\frac{1}{k}$ - наибольшее из строго убьвающих слагаемых, то $\frac{1}{k}>\frac{1}{5}$, т.е. $k=3$ или $k=4$.

Аналогично, при разбиении $4=2\left(a_{1}+a_{3}\right)+\left(a_{2}+a_{4}\right)+\left(a_{2}+a_{5}\right)+\left(a_{4}+a_{5}\right)$ получаем, что $w=3$ или $w=4$. Так как $k<w$, то $k=3, w=4$ и $\frac{1}{2}=\frac{1}{t}+\frac{1}{p}+\frac{1}{\tau}, \frac{1}{3}=\frac{1}{m}+\frac{1}{u}+\frac{1}{\tau}$.

Пользуясь леммой 4 и тем, что наименьшее слагаемое в двух последних уравнениях равно $\frac{1}{l}$, получаем, что общий знаменатель чисел $\frac{1}{t}, \frac{1}{p}, \frac{1}{t}, \frac{1}{m}$ и $\frac{1}{u}$ не превосходит 42 . Выражая $a_{3}, a_{4}, a_{5}$ и $a_{2}, a_{4}, a_{5}$ через эти числа и $k$ и $w$, получаем, что общий знаменатель чисел $a_{2}, a_{3}, a_{4}$ и $a_{5}$ не превосходит 84 . Но тогда и $q_{1} \leqslant 84$.

\section{СПИСОК ЛИТЕРАТУРЫ}

[1] Deligne P., Mostow G.D. // Pabl. Math. I.H.E.S. 1986. V. 63. P. 5-90. [2] Mostow G. D. // J. Amer. Math. Soc. 1988. V. 1. №3. P. 555-586. [3] Mostow G. D. // Publ. Math. I.H.E.S. 1986. V. 63. P. 91-106. [4] Thurston W. P. Shapes of polihedra // Preprint: Princeton I.A.S., 1987, June. [5] Weber M. Dissertation. Bonn. Math. Schriften, 1993. 